\title{
Prospective associations between the nutritional quality of foods consumed (graded by the FSAm-NPS underlying the Nutri-Score) and mortality in Europe
}

\author{
Mélanie Deschasaux $^{1,2}$, Inge Huybrechts ${ }^{3}$, Neil Murphy ${ }^{3}$, Chantal Julia ${ }^{2,4}$, Serge Hercberg ${ }^{2,4}$, \\ Bernard Srour $^{2}$, Emmanuelle Kesse-Guyot ${ }^{2}$, Paule Latino-Martel ${ }^{2}$, Carine Biessy ${ }^{3}$, \\ Corinne Casagrande ${ }^{3}$, Mazda Jenab ${ }^{3}$, Heather Ward ${ }^{5}$, Elisabete Weiderpass ${ }^{6}$, Pietro Ferrari ${ }^{3}$, \\ Elio Riboli ${ }^{5}$, Marc Gunter ${ }^{3}$ and Mathilde Touvier ${ }^{2}$ \\ ${ }^{1}$ on behalf of all EPIC collaborators, Bobigny, France, \\ ${ }^{2}$ Nutritional Epidemiology Research Team (EREN), Sorbonne Paris Cité Epidemiology and Statistics Research \\ Center, U1153 Inserm, U1125 Inra, Cnam, Paris 13 University, Bobigny, France, \\ ${ }^{3}$ Nutrition and Metabolism Section, International Agency for Research on Cancer, Lyon, France, \\ ${ }^{4}$ Public Health Department, Avicenne Hospital (AP-HP), Bobigny, France, \\ ${ }^{5}$ Faculty of Medicine, School of Public Health, Imperial College London, London, United Kingdom and \\ ${ }^{6}$ Department of Medical Epidemiology and Biostatistics, Karolinska Institutet, Stockholm, Sweden
}

\begin{abstract}
In 2017, 11 million deaths related to non-communicable diseases (NCDs) were attributable to dietary risk factors (GBD, 2019). Helping consumers make healthier food choices hence appears as one key strategy to prevent NCDs-related mortality. To this end, political authorities are considering implementing a simple label to reflect the nutritional quality of food products. The five-colour Nutri-Score label, derived from the Nutrient Profiling System of the British Food Standards Agency (FSAm-NPS), has been chosen by several countries in Europe (France, Belgium, Spain). Yet, its implementation is still voluntary per EU labelling regulation. Scientific evidence is therefore needed regarding the relevance of the FSAm-NPS at the European level. Following on our results showing an increased risk of cancer related to the consumption of foods with a high FSAm-NPS score in the EPIC cohort, our objective is now to focus on NCDs-related mortality. Our prospective analyses included 501,594 adults from the EPIC cohort (1992-2015, median follow-up: 17.2 years). Mortality events occurring $<2$ years after recruitment were excluded, leaving 50,743 death events (main causes: cancer, $\mathrm{n}=21,971$; cerebro/cardiovascular diseases, $\mathrm{n}=12,407$; respiratory diseases, $\mathrm{n}=2,796$ ). Usual food intakes were assessed with standardized country-specific diet assessment methods. The FSAm-NPS was calculated for each food/beverage using their 100-g content in energy, sugar, saturated fatty acid, sodium, fibres, proteins, and fruits/vegetables/ legumes/nuts. The individual FSAm-NPS Dietary Index (DI) is obtained as an energy-weighted mean of the FSAm-NPS scores of all food items usually consumed by a participant. Cox proportional hazards models adjusted for confounding factors, including personal history of cancer, cardiovascular diseases and diabetes were computed. Fine and Gray models were also tested to take into account competing events for cause-specific mortality analyses. A higher FSAm-NPS DI score, reflecting a lower nutritional quality of the diet, was associated with a higher mortality risk overall $\left(\mathrm{HR}_{\mathrm{Q} 5 \mathrm{vs} . \mathrm{Q} 1}=1.06[95 \% \mathrm{CI}\right.$ : $1.02-1.09]$, P-trend $\left.<0.001\right)$ and by cancer $\left(\mathrm{HR}_{\mathrm{Q} 5 \mathrm{vs.Q1}}=1.06\right.$ [1.01-1.11], P-trend $\left.=0.003\right)$ and respiratory diseases $\left(\mathrm{HR}_{\mathrm{Q} 5 \mathrm{vs.Q1}}=1.33\right.$ [1.16-1.52], P-trend $\left.<0.001\right)$, with similar results in competing events analyses. Associations with cerebro-/cardiovascular diseases mortality were weaker $\left(\mathrm{HR}_{\mathrm{Q} 5 \mathrm{vs} . \mathrm{Q} 1}=1.05\right.$ $[0.98,1.11]$, P-trend =0.04) and no longer statistically significant in competing events analyses. In this large multinational European cohort, the consumption of food products with a higher FSAm-NPS score (lower nutritional quality of the foods consumed) was associated with a higher mortality risk, supporting the relevance of the FSAm-NPS to grade the nutritional quality of food products for public health applications (e.g, Nutri-Score) aiming to guide the consumers towards healthier food choices.
\end{abstract}

\section{Conflict of Interest}

There is no conflict of interest 